in vivo $35: 507-515(2021)$

doi:10.21873/invivo.12285

\title{
A Prognostic Score for Predicting Survival in Patients With Pancreatic Head Adenocarcinoma and Distal Cholangiocarcinoma
}

\author{
EDOARDO MARIA MUTTILLO ${ }^{1}$, ANTONIO CIARDI $^{2}$, PAOLINA SAULLO $^{1}$, RAFFAELE TROIANO $^{1}$, \\ GABRIELE MASSELLI ${ }^{2}$, MARIANNA GUIDA $^{2}$, ALESSANDRA TORTORA $^{2}$, \\ ISABELLA SPERDUTI $^{3}$, GIULIO MARINELLO ${ }^{1}$, PIERO CHIRLETTI ${ }^{1}$ and ROBERTO CARONNA ${ }^{1}$ \\ ${ }^{1}$ Department of Surgical Sciences, Sapienza University of Rome, Rome, Italy; \\ ${ }^{2}$ Department of Radiological Sciences, Oncology and Pathology, Sapienza University of Rome, Rome, Italy; \\ ${ }^{3}$ Biostatistical Unit - Clinical Trials Center, IRCCS Regina Elena National Cancer Institute, Rome, Italy
}

\begin{abstract}
Background/Aim: Survival of patients with pancreatic cancer remains poor despite improvements in therapeutic strategies. This study aims to create a novel preoperative score to predict prognosis in patients with tumors of the pancreaticobiliary head. Patients and Methods: Data on 190 patients who underwent to pancreaticoduodenectomy at Sapienza University of Rome from January 2010 to December 2018 were retrospectively analyzed. After exclusion criteria, 101 patients were considered eligible for retrospective study. Preoperative biological, clinical and radiological parameters were considered. Results: Pancreatic ductal adenocarcinoma [hazard ratio $(H R)=1.995,95 \%$ confidence intervaI $(C I)=1.1-3.3 ; p=0.01]$, carbohydrate antigen 19.9 (CA 19.9) $>230 \mathrm{U} / \mathrm{ml}(\mathrm{HR}=2.414,95 \% \mathrm{CI}=2.4-1.5, p<0.0001)$ and Wirsung duct diameter $>3 \mathrm{~mm}$ (HR=1.592, 95\% CI=1.5-0.9; $p=0.08$ ) were the only parameters associated with poor prognosis. Through these parameters, a prognostic score (PHT score) was developed which predicted worst survival when exceeding 2 and better survival when $\leq 2$. Conclusion: The PHT score may have a potential impact on predicting overall survival and consequently modulate the timing and type of treatment (up-front surgery vs. neoadjuvant therapy) patients are offered.
\end{abstract}

This article is freely accessible online.

Correspondence to: Professor Roberto Caronna, Department of Surgical Sciences, Sapienza University of Rome, Viale Regina Elena 324, 00161 Rome, Italy. E-mail: roberto.caronna@uniroma1.it

Key Words: Pancreatic cancer, distal cholangiocarcinoma, pancreaticoduodenectomy, borderline resectable pancreatic cancer, prognostic score, survival.
Five-year survival of patients with pancreatic head tumors (PHT) still remains one of the greatest challenges in recent decades in the surgical/oncological field. In fact, although postoperative mortality is decreasing, especially in highvolume centers, 5-year survival has improved less significantly (1-3). Thus, the assessment of actual results and the role of surgery is hampered by a number of difficulties related to biology, staging and phenotypic tumor typing. Morphological investigation often proves inadequate for defining tumor biology correctly and justifying different evolution or responsiveness to adjuvant therapy of macroscopically similar tumors. In addition, the preoperative differential diagnosis between pancreatic ductal adenocarcinoma (PDAC) and distal cholangiocarcinoma (DC) remains an unresolved problem because differential diagnosis is often performed only after surgical resection $(4,5)$. This aspect greatly influences the long-term results, the two neoplasms having extremely different evolution and prognosis but often being treated as a single entity $(6,7)$. To date, the decision about the type of treatment to use for these neoplasms has always been guided by radiological decision, based on criteria that divide the neoplasms into resectable, borderline resectable and unresectable $(8,9)$. However, given the unsatisfactory results in PDAC in terms of long-term survival, new parameters associated with radiological parameters such as resectability must be found, whilst the use of neoadjuvant therapies is increasing even in patients with borderline resectable or resectable tumors (10).

In this scenario, some preoperative prognostic parameters may be useful for stratifying patients according to expected survival and for deciding which patients should be considered for upfront surgery or neoadjuvant chemotherapy. This study aimed to create a prognostic score, composed exclusively of pre-operative parameters, which allows prediction of the prognosis of patients with pancreatic head 
tumors (PHT), and, through that, to identify which patients are suitable for up-front surgical resection and which for neoadjuvant chemotherapy.

\section{Patients and Methods}

Patient selection and areas of study. For this retrospective study 190 consecutive patients who underwent pancreaticoduodenectomy at Sapienza University of Rome from January 2010 to December 2018 were analyzed. The inclusion criteria were diagnosis of pancreatic or biliary cancer obtained by definitive postoperative histological examination. Out of them, 89 cases with diagnosis of ampullary carcinoma, non ampullary duodenal adenocarcinoma, pancreatic metastasis, stage IV cancer and benign disease were excluded. Patients with jaundice with a high level of carbohydrate antigen 19.9 (CA19.9) were also excluded to prevent influence on marker validation.

Patients for whom follow-up was lost and patients who died due to surgical complications were excluded. In total 101 patients, divided into 66 with PDAC and 35 with DC were enrolled. In this retrospective study, all patients underwent up-front surgery.

Long-term follow-up was considered for all patients. Preoperative parameters were divided into three groups: Clinical, biological and radiological. The clinical parameters included gender, age, American Society of Anesthesiologists (ASA) score and body mass index (BMI) ( $>25 \mathrm{~kg} / \mathrm{m}^{2}$ as cut-off for being overweight). The biological parameters commonly examined preoperatively (analyzed within 1 month before surgery) were serum CA19.9 level, nutritional and inflammatory markers such as albumin, C-reactive protein (CRP), modified Glasgow prognostic score, neutrophil lymphocyte ratio, and platelet lymphocyte ratio. For each parameter, we considered cut-off values according to literature (11-15). Radiological parameters included Wirsung duct diameter measured at greatest dilation, pancreatic density and Pancreatic Attenuation Index (PAI) as the ratio of pancreatic and splenic density. As reported elsewhere, a Wirsung duct exceeding 3 $\mathrm{mm}$ was considered dilatated (16). Mean density values of pancreas and spleen were calculated in basic conditions; values were calculated automatically by system positioning on a region of interest where the parenchyma was most represented at computed tomographic scan. Density values $\geq 40 \mathrm{HU}$ were considered as high density and $<40 \mathrm{HU}$ as low density; with average ratio for pancreas/spleen of $<0.54$ as low and 0.54 as high, respectively $(16,17)$.

Statistical analysis. Descriptive statistics were used to summarize pertinent study information. Associations between categorical variables were analyzed according to the Pearson chi-square test or Fisher exact test, when appropriate.

The odds ratio (OR), hazard ratio (HR) and the $95 \%$ confidence intervals $(95 \% \mathrm{CI})$ were estimated using logistic regression and Cox regression univariate model. A multivariate logistic regression and proportional hazard model were developed using stepwise regression (forward selection, enter limit and remove limit, $p=0.10$ and $p=0.15$, respectively) to identify independent predictors of outcomes. The assessment of interactions between significant investigational variables was taken into account when developing the multivariate model.

Overall survival (OS) was estimated by the Kaplan-Meier product limit method from the date the surgical resection until death both for cancer and other causes. Curves were reported for those prognostic factors that were found to have independent influence at the multivariate analysis. The log-rank test was used to assess
Table I. General features of study patients.

\begin{tabular}{lc}
\hline Parameter & Category \\
\hline Gender, n (\%) & \\
$\quad$ Male & $62(61.4)$ \\
Female & $39(38.6)$ \\
Age, years & $69(44-87)$ \\
$\quad$ Mean (range) & $38(37.6)$ \\
BMI, n (\%) & $63(62.4)$ \\
$>25 \mathrm{kgm}^{2}$ & \\
$\quad 25 \mathrm{~kg} / \mathrm{m}^{2}$ & $66(65.3)$ \\
Primary tumor, n (\%) & $35(34.7)$ \\
PDAC & \\
DC & $85(84.16)$ \\
Resectability, n (\%) & $16(15.84)$ \\
Resectable & \\
Borderline & $8(7.9)$ \\
ASA, n (\%) & $59(58.4)$ \\
I & $33(32.7)$ \\
II & $1(1)$ \\
III & \\
IV &
\end{tabular}

ASA: American Society of Anesthesiologists; BMI: body mass index; DC: distal cholangiocarcinoma; PDAC: pancreatic ductal adenocarcinoma.

differences between subgroups. Significance was defined at a $p$ value of less than 0.05 .

The log-OR and $\log$-HR values obtained from the multivariate model were used to derive weighting factors for a continuous prognostic index, aimed at identifying differential risks of outcomes. Coefficient estimates were 'normalized' by dividing by the smallest one and rounding the resulting ratios to the nearest integer (18). Thus, a continuous score assigning patients an 'individualized' risk was generated. The score was dichotomized according to prognosis with receiver operating characteristic (ROC) analysis for the best cut-off point (19).

To address the multivariate model overfit and to validate the results, a cross-validation technique evaluating the reproducibility of the final multivariate model in predicting all outcomes was also investigated using a re-sampling procedure (20). This technique generates a number of simulation datasets (at least 100, each approximately $80 \%$ of the original size) by randomly selecting patients from the original sample to establish the consistency of the model across less-powered patient samples. Risk classes were generated on the basis of the combination of the risk factors found.

The ROC analysis allowed the predictive accuracy of the prognostic mode to be assessed by the determination of the area under the curve (21).

SPSS (version 21.0; IBM, Armonk, NY, USA) and MedCalc (version 14.2.1; MedCalc software, Ostend, Belgium) licensed statistical programs were used for all analyses.

\section{Results}

The study group (101 patients) was composed of 62 males and 39 females, with a mean age of 69 (range=44-87) years. There were $66(65.3 \%)$ patients with PDAC, while there 
Table II. Univariate and multivariate (Cox regression) analysis.

\begin{tabular}{|c|c|c|c|c|c|c|c|}
\hline \multirow{2}{*}{\multicolumn{2}{|c|}{ Preoperative factor }} & & & \multirow{2}{*}{$\frac{\text { Univariate }}{p \text {-Value }}$} & \multicolumn{3}{|c|}{ Multivariate } \\
\hline & & & & & HR & $95 \% \mathrm{CI}$ & $p$-Value* \\
\hline \multirow[t]{10}{*}{ Clinical } & Age & Mean \pm SD & $69(8.78)$ & & & & \\
\hline & & $\leq 69$ & 39 (38.7) & 0.16 & & & \\
\hline & & $>69$ & $62(61.3)$ & & & & \\
\hline & ASA & Mean \pm SD & $2.26 \pm 0.6$ & & & & \\
\hline & & $\leq 2$ & $67(66.3)$ & 0.29 & & & \\
\hline & & $>2$ & $34(33.7)$ & & & & \\
\hline & Gender & Male & $62(61.4)$ & 0.53 & & & \\
\hline & & Female & 39 (38.6) & & & & \\
\hline & BMI, $\mathrm{kg} / \mathrm{m}^{2}$ & $\leq 25$ & $38(37.6)$ & 0.96 & & & \\
\hline & & $>25$ & $63(62.4)$ & & & & \\
\hline \multirow[t]{14}{*}{ Biological } & Primary & PDAC & $66(65.3)$ & $<0.0001$ & 1.995 & $1.1-3.3$ & 0.01 \\
\hline & & $\mathrm{DC}$ & $35(34.7)$ & & & & \\
\hline & Albumin, g/1 & $\geq 35$ & $50(49.5)$ & 0.31 & & & \\
\hline & & $<35$ & $51(50.5)$ & & & & \\
\hline & $\mathrm{CRP}, \mathrm{mg} / \mathrm{dl}$ & $\leq 10$ & $54(53.5)$ & 0.16 & & & \\
\hline & & $>10$ & $47(46.5)$ & & & & \\
\hline & mGPS & $0-1$ & $66(65.3)$ & 0.03 & & & n.s. \\
\hline & & 2 & $35(34.7)$ & & & & \\
\hline & NLR & $\leq 2.7$ & $43(42.5)$ & 0.87 & & & \\
\hline & & $>2.7$ & $58(57.5)$ & & & & \\
\hline & PLR & $\leq 146$ & $43(42.5)$ & 0.80 & & & \\
\hline & & $>146$ & $58(57.5)$ & & & & \\
\hline & CA19.9, U/ml & $\leq 230$ & $49(48.5)$ & $<0.0001$ & 2.414 & $2.4-1.5$ & $<0.0001$ \\
\hline & & $>230$ & $52(51.5)$ & & & & \\
\hline \multirow[t]{6}{*}{ Radiological } & Wirsung duct, mm & $\leq 3$ & $34(33.6)$ & 0.001 & 1.592 & $1.5-0.9$ & 0.082 \\
\hline & & $>3$ & $67(66.4)$ & & & & \\
\hline & Pancreatic density & $\leq 40 \mathrm{HU}$ & $78(77.2)$ & 0.06 & & & \\
\hline & & $>40 \mathrm{HU}$ & $23(22.8)$ & & & & \\
\hline & PAI & $<0.54$ & $35(34.6)$ & 0.60 & & & \\
\hline & & $\geq 0.54$ & $66(65.4)$ & & & & \\
\hline
\end{tabular}

ASA: American Society of Anesthesiologists; BMI: body mass index; CA 19.9: carbohydrate antigen 19.9; CI: confidence interval; CRP: C-reactive protein; DC: distal cholangiocarcinoma; HR: hazard ratio; mGPS: modified Glasgow Prognostic Score; NLR: neutrophil-lymphocyte ratio ; PAI: pancreatic attenuation index; PDAC: pancreatic ductal adenocarcinoma; PLR: platelet-lymphocyte ratio; SD: standard deviation. *A multivariate logistic regression and proportional hazard model were developed using stepwise regression (forward selection, enter and remove limits, $p=0.10$ and $p=0.15$, respectively). Statistically significant $p$-values are shown in bold. N.s.: Not significant.

were $35(34.7 \%)$ with DC. There were 38 patients with a BMI greater than $>25 \mathrm{~kg} / \mathrm{m}^{2}$. As reported in Table I, American Society of Anesthesiologists score of 2 and patients with resectable tumors were prevalent (8).

At multivariate analyses, the only parameters independently associated with an unfavorable prognosis were PDAC $(\mathrm{HR}=1.995,95 \% \mathrm{CI}=1.1-3.3 ; p=0.01), \mathrm{CA} 19.9>230 \mathrm{U} / \mathrm{ml}$ $(\mathrm{HR}=2.414,95 \% \mathrm{CI}=2.4-1.5 ; p<0.0001)$ and Wirsung duct diameter $>3 \mathrm{~mm}(\mathrm{HR}=1.592,95 \% \mathrm{CI}=1.5-0.9 ; p=0.082)$ as shown in Table II. At multivariate analyses, prognostic factors for worse survival were: PDAC $(\mathrm{OR}=2.159,95 \% \mathrm{CI}=0.8$ 25.3; $p=0.04)$ and $\mathrm{CA} 19.9>230 \mathrm{U} / \mathrm{ml}(\mathrm{OR}=9.253,95 \%$ $\mathrm{CI}=3.3-25.3 ; p<0.0001)$ at 1 year; PDAC $(\mathrm{OR}=3.560 ; 95 \%$ $\mathrm{CI}=1.2-10.4 ; p=0.02)$ age $(\mathrm{OR}=7.000,95 \% \mathrm{CI}=1.8-26.4$; $p=0.004)$ and pancreatic density $<40 \mathrm{HU}(\mathrm{OR}=3.043,95 \%$
$\mathrm{CI}=0.9-9.4 ; p=0.05)$ at 3 years; and finally only PDAC $(\mathrm{OR}=9.845,95 \% \mathrm{CI}=1.9-49.5 ; p=0.006)$ at 5 years, as shown in Tables III-V. Through the significant parameters for OS, a prognostic score has been developed for stratify patients according to the risk of poor prognosis.

Points were assigned for the PHT score ranged between 0 and 4 points as shown in Table VI. Using a cut-off PHT score of 2 as estimated through the ROC curve, when the score exceeded 2, a poor prognosis after up-front surgical resection was predicted, while a better prognosis after surgical resection was predicted when the score was $\leq 2$ with high accuracy (area under the curve of 76\%).

Through the Kaplan-Meier estimator, survival of groups with PHT scores of $>2$ and $\leq 2$ were compared. The results showed survival of those with PHT score of 2 or less was 
Table III. Univariate and multivariate analysis for 1-year overall survival (OS).

\begin{tabular}{|c|c|c|c|c|c|c|c|}
\hline \multirow[b]{2}{*}{ Preoperative factor } & & & \multirow[b]{2}{*}{ OS, $\%$} & \multirow{2}{*}{$\frac{\text { Univariate }}{p \text {-Value }}$} & \multicolumn{3}{|c|}{ Multivariate } \\
\hline & & & & & HR & $95 \% \mathrm{CI}$ & $p$-Value* \\
\hline \multirow[t]{9}{*}{ Clinical } & Total cohort & Subgroup & & & & & \\
\hline & Age, years & $\leq 69$ & $74 \%$ & 0.48 & & & \\
\hline & & $>69$ & $49 \%$ & & & & \\
\hline & ASA & $\leq 2$ & $62.6 \%$ & 0.31 & & & \\
\hline & & $>2$ & $50 \%$ & & & & \\
\hline & Gender & Male & $59.7 \%$ & 0.94 & & & \\
\hline & & Female & $56.4 \%$ & & & & \\
\hline & BMI, $\mathrm{kg} / \mathrm{m}^{2}$ & $\leq 25$ & $57.1 \%$ & 0.85 & & & \\
\hline & & $>25$ & $60.5 \%$ & & & & \\
\hline \multirow[t]{14}{*}{ Biological } & Primary & PDAC & $48.8 \%$ & 0.03 & 2.159 & $0.8-25.3$ & 0.04 \\
\hline & & DC & $77 \%$ & & & & \\
\hline & Albumin, g/l & $\geq 35$ & $68 \%$ & 0.03 & & & n.s. \\
\hline & & $<35$ & $49 \%$ & & & & \\
\hline & $\mathrm{CRP}, \mathrm{mg} / \mathrm{dl}$ & $\leq 10$ & $70 \%$ & 0.01 & & & n.s. \\
\hline & & $>10$ & $44.6 \%$ & & & & \\
\hline & mGPS & $0-1$ & $68.1 \%$ & 0.005 & & & n.s. \\
\hline & & 2 & $40 \%$ & & & & \\
\hline & NLR & $\leq 2.7$ & $62.7 \%$ & 0.31 & & & \\
\hline & & $>2.7$ & $55.1 \%$ & & & & \\
\hline & PLR & $\leq 146$ & $58 \%$ & 0.82 & & & \\
\hline & & $>146$ & $58 \%$ & & & & \\
\hline & $\mathrm{CA} 19.9, \mathrm{U} / \mathrm{ml}$ & $\leq 230$ & $83.6 \%$ & $<0.0001$ & 9.253 & $3.3-25.3$ & $<0.0001$ \\
\hline & & $>230$ & $34.6 \%$ & & & & \\
\hline \multirow[t]{6}{*}{ Radiological } & Wirsung duct, mm & $\leq 3$ & $70 \%$ & 0.042 & & & n.s. \\
\hline & & $>3$ & $52.2 \%$ & & & & \\
\hline & Pancreatic density & $\leq 40 \mathrm{HU}$ & $56.4 \%$ & 0.51 & & & \\
\hline & & $>40 \mathrm{HU}$ & $65.2 \%$ & & & & \\
\hline & PAI & $<0.54$ & $57.1 \%$ & 0.73 & & & \\
\hline & & $\geq 0.54$ & $59 \%$ & & & & \\
\hline
\end{tabular}

ASA: American Society of Anesthesiologists; BMI: body mass index; CA 19.9: carbohydrate antigen 19.9; CI: confidence interval; CRP: C-reactive protein; DC: distal cholangiocarcinoma; HR: hazard ratio; MGPS: modified Glasgow Prognostic Score; NLR: neutrophil-lymphocyte ratio ; PAI: pancreatic attenuation index; PDAC: pancreatic ductal adenocarcinoma; PLR: platelet-lymphocyte ratio; SD: standard deviation. *A multivariate logistic regression and proportional hazard model were developed using stepwise regression (forward selection, enter and remove limits, $p=0.10$ and $p=0.15$, respectively). Statistically significant $p$-values are shown in bold. N.s.: Not significant.

significantly better $(p<0.0001$; Figure 1$)$. Figures $2-4$ compare survival results between patients according to disease subtype (PDAC vs. DC), CA19.9 level $>230 \mathrm{U} / \mathrm{ml}$ $v s . \leq 230 \mathrm{U} / \mathrm{ml}$, and Wirsung duct diameter $(\leq 3 v s .>3 \mathrm{~mm})$ to highlight their prognostic value.

\section{Discussion}

Despite recent improvements, PHT remains an extremely aggressive cancer with poor prognosis, even in those with resectable disease, with an OS rate at 5 years of only $10 \%$ (22). Surgical resection is the only curative treatment for PHT but only $20 \%$ of patients have resectable disease at the time of diagnosis (23). However, those same patients often have poor prognosis despite undergoing up-front resection due to the presence of micrometastases not detectable at the time of diagnosis. Recently, neoadjuvant chemotherapy has been a major research focus for improving the outcome for PHT (24). Although its role still needs to be clarified, it has shown some advantages in term of $\mathrm{R} 0$ resection, even in patients with resectable and borderline resectable tumors $(25,26)$. It is not yet clear which patients can really benefit from neoadjuvant treatment. Often the diagnosis between PDAC and DC is obtained only with histological postoperative examination, due to their similar histological pattern (both adenocarcinoma) which does not allow them to be easily distinguished in preoperative (echoendoscopic/percutaneous) biopsy (5). The difficulty in differential diagnosis may represent a bias in term of indications for targeted neoadjuvant chemotherapy especially in those with borderline resectable tumors (risk of non-target chemotherapy), where it is recommended in patients with PDAC according to the 2019 National 
Table IV. Univariate and multivariate analysis for 3-year overall survival (OS).

\begin{tabular}{|c|c|c|c|c|c|c|c|}
\hline \multirow{2}{*}{\multicolumn{3}{|c|}{ Preoperative factor }} & \multirow[b]{2}{*}{ OS, $\%$} & \multirow{2}{*}{$\frac{\text { Univariate }}{p \text {-Value }}$} & \multicolumn{3}{|c|}{ Multivariate } \\
\hline & & & & & $\mathrm{HR}$ & $95 \% \mathrm{CI}$ & $p$-Value* \\
\hline \multirow[t]{9}{*}{ Clinical } & Total cohort & Subgroup & & & & & \\
\hline & Age, years & $\leq 69$ & $30 \%$ & 0.04 & 7.000 & $1.8-26.4$ & 0.004 \\
\hline & & $>69$ & $14 \%$ & & & & \\
\hline & ASA & $\leq 2$ & $23.8 \%$ & 0.12 & & & \\
\hline & & $>2$ & $14.7 \%$ & & & & \\
\hline & Gender & Male & $16.1 \%$ & 0.14 & & & \\
\hline & & Female & $28.2 \%$ & & & & \\
\hline & BMI, $\mathrm{kg} / \mathrm{m} 2$ & $\leq 25$ & $22.2 \%$ & 0.49 & & & \\
\hline & & $>25$ & $18.4 \%$ & & & & \\
\hline \multirow[t]{14}{*}{ Biological } & Primary & PDAC & $15.5 \%$ & 0.01 & 3.560 & $1.2-10.4$ & 0.021 \\
\hline & & DC & $31.4 \%$ & & & & \\
\hline & Albumin, g/l & $\geq 35$ & $24 \%$ & 0.19 & & & \\
\hline & & $<35$ & $17.6 \%$ & & & & \\
\hline & $\mathrm{CRP}, \mathrm{mg} / \mathrm{dl}$ & $\leq 10$ & $24 \%$ & 0.39 & & & \\
\hline & & $>10$ & $17 \%$ & & & & \\
\hline & mGPS & $0-1$ & $25.7 \%$ & 0.08 & & & \\
\hline & & 2 & $4 \%$ & & & & \\
\hline & NLR & $\leq 2.7$ & $16.2 \%$ & 0.75 & & & \\
\hline & & $>2.7$ & $24.1 \%$ & & & & \\
\hline & PLR & $\leq 146$ & $16.2 \%$ & 0.75 & & & \\
\hline & & $>146$ & $24.1 \%$ & & & & \\
\hline & CA19.9, U/ml & $\leq 230$ & $83.6 \%$ & 0.02 & & & n.s. \\
\hline & & $>230$ & $34.6 \%$ & & & & \\
\hline \multirow[t]{6}{*}{ Radiological } & Wirsung duct, mm & $\leq 3$ & $26.4 \%$ & 0.08 & & & \\
\hline & & $>3$ & $17.9 \%$ & & & & \\
\hline & Pancreatic density & $\leq 40 \mathrm{HU}$ & $15.3 \%$ & 0.01 & 3.043 & $0.9-9.4$ & 0.053 \\
\hline & & $>40 \mathrm{HU}$ & $39.1 \%$ & & & & \\
\hline & PAI & $<0.54$ & $17 \%$ & 0.36 & & & \\
\hline & & $\geq 0.54$ & $22.7 \%$ & & & & \\
\hline
\end{tabular}

ASA: American Society of Anesthesiologists; BMI: body mass index; CA 19.9: carbohydrate antigen 19.9; CI: confidence interval; CRP: C-reactive protein; DC: distal cholangiocarcinoma; HR: hazard ratio; mGPS: modified Glasgow Prognostic Score; NLR: neutrophil-lymphocyte ratio ; PAI: pancreatic attenuation index; PDAC: pancreatic ductal adenocarcinoma; PLR: platelet-lymphocyte ratio; SD: standard deviation. *A multivariate logistic regression and proportional hazard model were developed using stepwise regression (forward selection, enter and remove limits, $p=0.10$ and $p=0.15$, respectively). Statistically significant $p$-values are shown in bold. N.s.: Not significant.

Comprehensive Cancer Network guidelines (27). This aspect is more important in patients with DC, where non target neoadjuvant chemotherapy may allow disease progression, especially in those with resectable tumors. Some authors have tried to stratify patients with pancreatic cancer based on prognostic risk factors, creating predictive models of unfavorable prognosis, which may help to select the patients correctly and eventually modulate postoperative treatments. However, most of these are postoperative models that allow prediction of prognosis only after surgical resection (28). Only Balzano et al. have developed a preoperative predictive model of survival after surgical resection, considering a large number of preoperative factors but only 1-year mortality outcome, and no radiological findings were considered (29).

Our study analyzed a large number of exclusively preoperative parameters in order to obtain a truly preoperative predictive model of poor survival that allows the best treatment to be selected for patients. To obtain a preoperative predictive model, a score was developed which includes three parameters: PDAC, Wirsung duct $>3 \mathrm{~mm}$ and CA $19.9>230 \mathrm{U} / \mathrm{ml}$. PDAC was also found to be the only significant factor for an unfavorable prognosis based on OS at 1,3 and 5 years. Thus, it is important to obtain data preoperatively, despite the difficulties outlined above. A Wirsung duct $>3 \mathrm{~mm}$ represents an innovative finding in terms unfavorable prognosis, since it has always been considered a factor associated with low incidence of pancreatic fistula. This finding might be explained by a greater development of exocrine pancreatic insufficiency and related malnutrition (30-32).

Similarly in regard to pancreatic density, we assumed that a low pancreatic density $(<40 \mathrm{HU})$ may allow local cancer 
Table V. Univariate and multivariate analysis for 5-year overall survival (OS).

\begin{tabular}{|c|c|c|c|c|c|c|c|}
\hline \multirow[b]{2}{*}{ Preoperative factor } & & & \multirow[b]{2}{*}{ OS, $\%$} & \multirow{2}{*}{$\frac{\text { Univariate }}{p \text {-Value }}$} & \multicolumn{3}{|c|}{ Multivariate } \\
\hline & & & & & $\mathrm{HR}$ & $95 \% \mathrm{CI}$ & $p$-Value* \\
\hline \multirow[t]{9}{*}{ Clinical } & Total cohort & Subgroup & & & & & \\
\hline & Age, years & $\leq 69$ & $7.6 \%$ & 0.76 & & & \\
\hline & & $>69$ & $6.5 \%$ & & & & \\
\hline & ASA & $\leq 2$ & $7.4 \%$ & 0.12 & & & \\
\hline & & $>2$ & $5.8 \%$ & & & & \\
\hline & Gender & Male & $4.8 \%$ & 0.45 & & & \\
\hline & & Female & $10.2 \%$ & & & & \\
\hline & BMI, $\mathrm{kg} / \mathrm{m} 2$ & $\leq 25$ & $7.9 \%$ & 0.63 & & & \\
\hline & & $>25$ & $5.2 \%$ & & & & \\
\hline \multirow[t]{14}{*}{ Biological } & Primary & PDAC & $3 \%$ & 0.006 & 9.845 & $1.9-49.5$ & 0.006 \\
\hline & & $\mathrm{DC}$ & $14.5 \%$ & & & & \\
\hline & Albumin, g/1 & $\geq 35$ & $4 \%$ & 0.64 & & & \\
\hline & & $<35$ & $9.8 \%$ & & & & \\
\hline & $\mathrm{CRP}, \mathrm{mg} / \mathrm{dl}$ & $\leq 10$ & $9.2 \%$ & 0.55 & & & \\
\hline & & $>10$ & $4.25 \%$ & & & & \\
\hline & mGPS & $0-1$ & $7.5 \%$ & 0.63 & & & \\
\hline & & 2 & $5.7 \%$ & & & & \\
\hline & NLR & $\leq 2.7$ & $0 \%$ & 0.15 & & & \\
\hline & & $>2.7$ & $12.6 \%$ & & & & \\
\hline & PLR & $\leq 146$ & $2.3 \%$ & 0.89 & & & \\
\hline & & $>146$ & $10.3 \%$ & & & & \\
\hline & $\mathrm{CA} 19.9, \mathrm{U} / \mathrm{ml}$ & $\leq 230$ & $8.1 \%$ & 0.15 & & & \\
\hline & & $>230$ & $5.6 \%$ & & & & \\
\hline \multirow[t]{6}{*}{ Radiological } & Wirsung duct, mm & $\leq 3$ & $11.7 \%$ & 0.01 & & & n.s. \\
\hline & & $>3$ & $4.4 \%$ & & & & \\
\hline & Pancreatic density & $\leq 40 \mathrm{HU}$ & $3.8 \%$ & 0.04 & & & n.s. \\
\hline & & $>40 \mathrm{HU}$ & $17.3 \%$ & & & & \\
\hline & PAI & $<0.54$ & $5.7 \%$ & 0.33 & & & \\
\hline & & $\geq 0.54$ & $7.5 \%$ & & & & \\
\hline
\end{tabular}

ASA: American Society of Anesthesiologists; BMI: body mass index; CA 19.9: carbohydrate antigen 19.9; CI: confidence interval; CRP: C-reactive protein; DC: distal cholangiocarcinoma; HR: hazard ratio; mGPS: modified Glasgow Prognostic Score; NLR: neutrophil-lymphocyte ratio ; PAI: pancreatic attenuation index; PDAC: pancreatic ductal adenocarcinoma; PLR: platelet-lymphocyte ratio; SD: standard deviation. *A multivariate logistic regression and proportional hazard model were developed using stepwise regression (forward selection, enter and remove limits, $p=0.10$ and $p=0.15$, respectively). Statistically significant $p$-values are shown in bold. N.s.: Not significant.

spread; however, this hypothesis was only partially confirmed because pancreatic density $<40 \mathrm{UH}$ was significantly related to an unfavorable prognosis only at 3 years (Table IV).

We confirmed that a high serum CA19.9 level is an important factor predictive of poor survival, as already highlighted by others, and a significant marker of locally advanced tumor and cancer recurrence (11, 33-35).

The PHT score might be useful to select patients not eligible for up-front surgery for whom neoadjuvant therapy should be considered, given the expected poor prognosis suggested by a PHT score $>2$ (Table VI). This approach allows neoadjuvant therapy to be also considered in patients with resectable disease with expected poor prognosis and with high risk of $\mathrm{R} 1$ resection to make surgery feasible (36). Patients with Wirsung duct $<3 \mathrm{~mm}$, CA19.9 $\leq 230 \mathrm{U} / \mathrm{ml}$ and supposed DC (PHT score <2) should be considered for up-front surgery.
Table VI. Pancreatic head tumor (PHT) prognostic score.

\begin{tabular}{lllc}
\hline & & \multicolumn{2}{c}{ PHT score } \\
\cline { 3 - 4 } Factor & Subgroup & Beta & Score \\
\hline Primary tumor & PDAC vs. DC & 0.690 & 1 \\
Wirsung duct & $>3$ vs. $\leq 3 \mathrm{~mm}$ & 0.465 & 1 \\
CA19.9 & $>230$ vs. $\leq 230 \mathrm{U} / \mathrm{ml}$ & 0.881 & 2 \\
\hline
\end{tabular}

PDAC: Pancreatic ductal adenocarcinoma; DC: distal cholangiocarcinoma.

This score presents some advantages: It is easily obtainable and feasible by routine biological and radiological parameters, does not requiring any advanced technological equipment and no additional cost. However, this study presents some limits: 


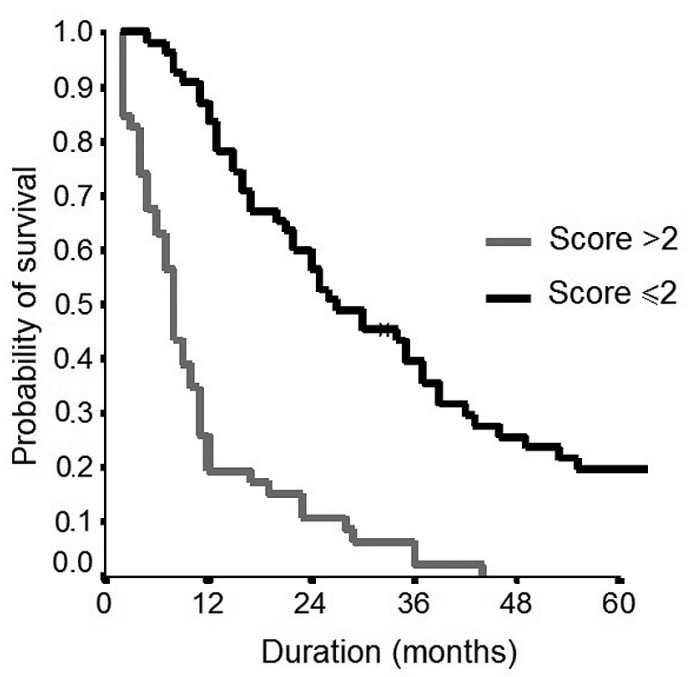

Figure 1. Overall survival according to pancreatic head tumor (PHT) score. Overall survival was significantly better in patients with $P H T \leq 2$ than those with PHT>2.

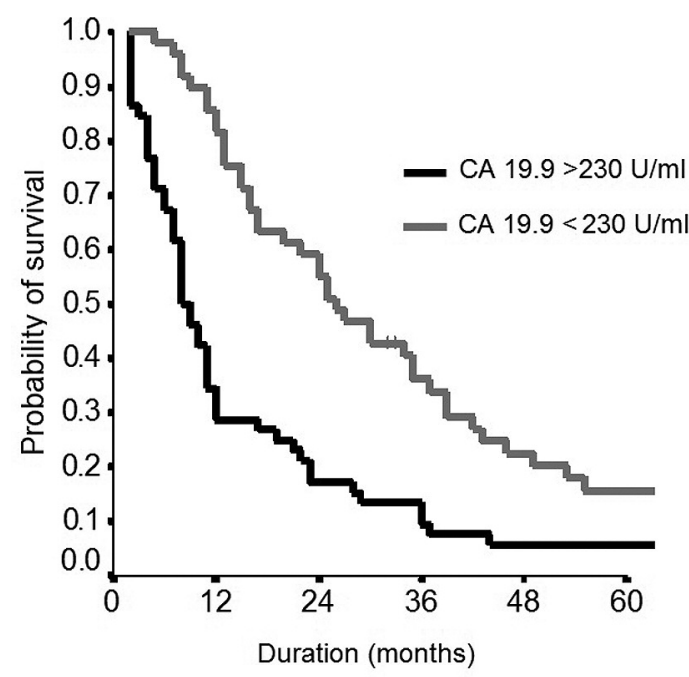

Figure 2. Overall survival according to serum level of carbohydrate antigen 19.9 (CA19.9).

It was a retrospective study and analyzed a limited sample of patients, although highly selected.

It would be useful to evaluate the prognostic accuracy of this score, applying it to a larger sample of patients undergoing neoadjuvant therapy and retrospectively evaluated on the definitive histological examination (postoperative). This is mainly to understand whether the best treatment to consider in patients with PDAC and DC with a score $>2$ but resectable disease is up-front surgery or neoadjuvant chemotherapy.

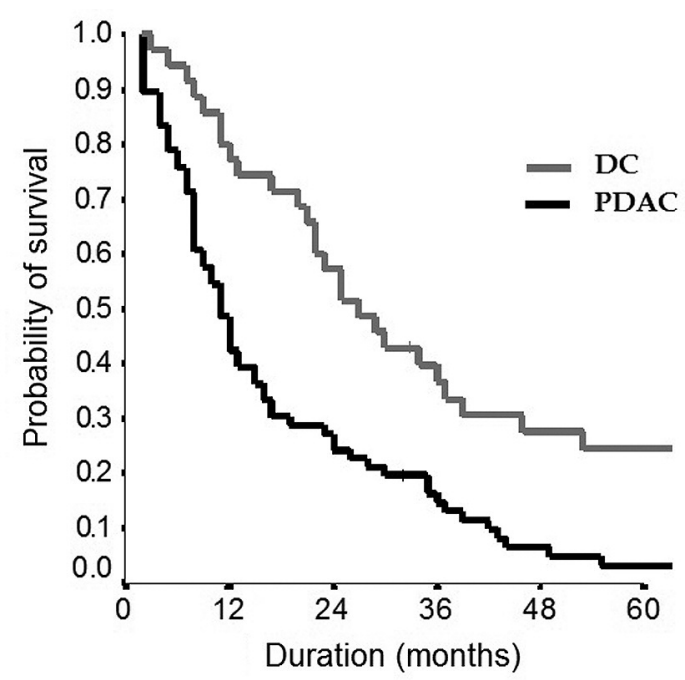

Figure 3. Overall survival according to primary tumor type. PDAC: Pancreatic ductal adenocarcinoma; DC: distal cholangiocarcinoma.

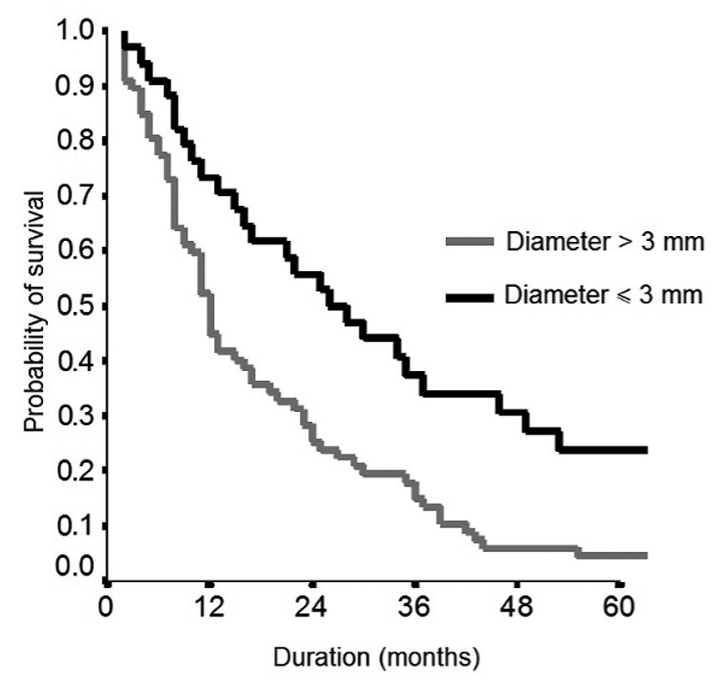

Figure 4. Overall survival according to the diameter of the Wirsung duct.

\section{Conclusion}

Better selection of patients who undergo pancreaticoduodenectomy is mandatory for obtaining better results. The use of neoadjuvant chemotherapy should be further evaluated in order to avoid unnecessary surgical resection and to select patients with responsive neoplasms for pancreaticoduodenectomy. The PHT score presented in this study is an innovative proposal, and is derived from entirely pre- 
operative parameters and is useful for making the decision as to whether patients should undergo up-front surgery or neoadjuvant therapy.

\section{Conflicts of Interest}

The Authors have no conflicts of interest to declare.

\section{Authors' Contributions}

All Authors participated in the study. CR conceived the idea for this study. CA carried out the histopathological examinations. MG, GM and TA performed the review of the radiological images and collected radiological data. MEM, MG, TR, specificity undertook the pre- and post-operative management of the patients and collected patient data. CP and CR performed most of the surgical procedures. MEM and SI performed data analysis. MEM and CR wrote the article. CR finalized the writing of the article. All Authors have read and accepted the contents of the article.

\section{Acknowledgements}

The Authors express special thanks to Professor Piero Chirletti for his precious teaching.

\section{References}

1 Stella M, Bissolati M, Gentile D and Arriciati A: Impact of surgical experience on management and outcome of pancreatic surgery performed in high- and low-volume centers. Updates Surg 69(3): 351-358, 2017. PMID: 28215039. DOI: 10.1007/s13304017-0422-3

2 Ho V and Heslin MJ: Effect of hospital volume and experience on in-hospital mortality for pancreaticoduodenectomy. Ann Surg 237(4): 509-514, 2003. PMID: 12677147. DOI: 10.1097/01.sla.00 00059981.13160 .97

3 Bray, F Ferlay J, Soerjomataram I, Siegel RL, Torre LA and Jemal A: Global cancer statistics 2018: GLOBOCAN estimates of incidence and mortality worldwide for 36 cancers in 185 countries. CA Cancer J Clin 68(6): 394-424, 2018. PMID: 30207593. DOI: $10.3322 /$ caac. 21492

4 Dumonceau JM, Deprez PH, Jenssen C, Iglesias-Garcia J, Larghi A, Vanbiervliet G, Aithal GP, Arcidiacono PG, Bastos P, Carrara S, Czakó L, Fernández-Esparrach G, Fockens P, Ginès À, Havre RF, Hassan C, Vilmann P, van Hooft JE and Polkowski M: Indications, results, and clinical impact of endoscopic ultrasound (EUS)-guided sampling in gastroenterology: European Society of Gastrointestinal Endoscopy (ESGE) Clinical Guideline Updated January 2017. Endoscopy 49(7): 695-714, 2017. PMID: 28511234. DOI: $10.1055 / \mathrm{s}-0043-109021$

5 Dumonceau JM, Polkowski M, Larghi A, Vilmann P, Giovannini M, Frossard JL, Heresbach D, Pujol B, FernándezEsparrach G, Vazquez-Sequeiros E and Ginès A: Indications, results, and clinical impact of endoscopic ultrasound (EUS)guided sampling in gastroenterology: European Society of Gastrointestinal Endoscopy (ESGE) Clinical Guideline. Endoscopy 43(10): 897-912, 2011. PMID: 21842456. DOI:10. 1055/s-0030-1256754
6 Zhou Y, Liu S, Wu L and Wan T: Survival after surgical resection of distal cholangiocarcinoma: A systematic review and meta-analysis of prognostic factors. Asian J Surg 40(2): 129-138, 2017. PMID: 26337377. DOI: 10.1016/j.asjsur.2015.07.002

7 Ethun CG, Lopez-Aguiar AG, Pawlik TM, Poultsides G, Idrees K, Fields RC, Weber SM, Cho C, Martin RC, Scoggins CR, Shen P, Schmidt C, Hatzaras I, Bentrem D, Ahmad S, Abbott D, Kim HJ, Merchant N, Staley CA, Kooby DA and Maithel SK: Distal cholangiocarcinoma and pancreas adenocarcinoma: are they really the same disease? A 13-institution study from the US Extrahepatic Biliary Malignancy Consortium and the Central Pancreas Consortium. J Am Coll Surg 224(4): 406-413, 2017. PMID: 28017812. DOI: 10.1016/j.jamcollsurg.2016.12.006

8 Bockhorn M, Uzunoglu FG, Adham M, Imrie C, Milicevic M, Sandberg AA, Asbun HJ, Bassi C, Büchler M, Charnley RM, Conlon K, Cruz LF, Dervenis C, Fingerhutt A, Friess H, Gouma DJ, Hartwig W, Lillemoe KD, Montorsi M, Neoptolemos JP, Shrikhande SV, Takaori K, Traverso W, Vashist YK, Vollmer C, Yeo CJ and Izbicki JR: Borderline resectable pancreatic cancer: A consensus statement by the International Study Group of Pancreatic Surgery (ISGPS). Surgery 155(6): 977-988, 2014. PMID: 24856119. DOI: 10.1016/j.surg.2014.02.001

9 Isaji S, Mizuno S, Windsor JA, Bassi C, Fernández-Del Castillo C, Hackert T, Hayasaki A, Katz MHG, Kim SW, Kishiwada M, Kitagawa $\mathrm{H}$, Michalski CW, Wolfgang CL: International consensus on definition and criteria of borderline resectable pancreatic ductal adenocarcinoma 2017. Pancreatology 18(1): 211, 2018. PMID: 29191513. DOI: 10.1016/j.pan.2017.11.011

10 Salvia R, Malleo G, Maggino L, Milella M and Bassi C: Pancreatic ductal adenocarcinoma: Time for a neoadjuvant revolution? Updates Surg 72(2): 321-324, 2020. PMID: 32445032. DOI:10.1007/s13304-020-00798-3

11 Asaoka T, Miyamoto A, Maeda S, Tsujie M, Hama N, Yamamoto K, Miyake M, Haraguchi N, Nishikawa K, Hirao M, Ikeda M, Sekimoto M and Nakamori S: Prognostic impact of preoperative NLR and CA19-9 in pancreatic cancer. Pancreatology 16(3): 434 440, 2016. PMID: 26852169. DOI: 10.1016/j.pan.2015.10.006

12 Wu LX, Wang XY, Xu KQ, Lin YL, Zhu WY, Han L, Shao YT, Zhou HY, Jiang H, Hang JJ and Yang XG: A systematic inflammation-based model in advanced pancreatic ductal adenocarcinoma. J Cancer 10(26): 6673-6680, 2019. PMID: 31777596. DOI: $10.7150 /$ jca.30561

13. Yamada S, Fujii T, Yabusaki N, Murotani K, Iwata N, Kanda M, Tanaka C, Nakayama G, Sugimoto H, Koike M, Fujiwara M and Kodera Y: Clinical implication of inflammation-based prognostic score in pancreatic cancer Glasgow prognostic score is the most reliable parameter. Medicine 95(18): e3582, 2016. PMID: 27149487. DOI: 10.1097/MD.0000000000003582

14 Glen P, Jamieson NB, McMillan DC, Carter R, Imrie CW and McKay CJ: Evaluation of an inflammation-based prognostic score in patients with inoperable pancreatic cancer. Pancreatology 6(5): 450-453, 2006. PMID: 16847382. DOI:10.1159/000094562

15 Hoshimoto S, Hishinuma S, Shirakawa H, Tomikawa M, Ozawa I and Ogata Y: Validation and clinical usefulness of pre- and postoperative systemic inflammatory parameters as prognostic markers in patients with potentially resectable pancreatic cancer. Pancreatology 20(2): 239-246, 2020. PMID: 31862230. DOI: 10.1016/j.pan.2019.12.004

16 Deng Y, Zhao B, Yang M, Li C and Zhang L: Association between the incidence of pancreatic fistula after pancreaticoduodenectomy 
and the degree of pancreatic fibrosis. J Gastrointest Surg 22(3): 438443, 2018. PMID: 29330723. DOI: 10.1007/s11605-017-3660-2

17 Yardimci S, Kara YB, Tuney D, Attaallah W, Ugurlu MU, Dulundu E and Yegen ŞC: A simple method to evaluate whether pancreas texture can be used to predict pancreatic fistula risk after pancreatoduodenectomy. J Gastrointest Surg 19(9): 1625-1631, 2015. PMID: 25982120. DOI:10.1007/s11605-015-2855-7

18 Di Maio M, Lama N, Morabito A, Smit EF, Georgoulias V, Takeda K, Quoix E, Hatzidaki D, Wachters FM, Gebbia V, Tsai CM, Camps C, Schuette W, Chiodini P, Piccirillo MC, Perrone F, Gallo C and Gridelli C: Clinical assessment of patients with advanced non-small-cell lung cancer eligible for second-line chemotherapy: A prognostic score from individual data of nine randomised trials. Eur J Cancer 46(4): 735-743, 2010. PMID: 20045311. DOI: 10.1016/j.ejca.2009.12.013

19 Penciana MJ and D'Agostino RB: Overall C as a measure of discrimination in survival analysis: Model specific population value and confidence interval estimation. Stat. Med 23(13): 2109-2123, 2004. PMID: 15211606. DOI: 10.1002/sim.1802

20 Sauerbrei $\mathrm{W}$ and Schumacher M: A bootstrap resampling procedure for model building: Application to the Cox regression model. Stat Med 11(16): 2093-2109, 1992. PMID: 1293671. DOI: $10.1002 / \operatorname{sim} .4780111607$

21 Hanley JA and McNeil BJ: The meaning and use of the area under a receiver operating characteristic (ROC) curve. Radiology 143(1): 29-36, 1982. PMID: 7063747. DOI: 10.1148/radiology.143.1.7063747

22 Siegel RL, Miller KD and Jemal A: Cancer statistics 2018. CA Cancer J Clin 68(1): 7-30, 2018. PMID: 29313949. DOI: $10.3322 /$ caac. 21442

23 Klaiber U, Leonhardt CS, Strobel O, Tjaden C, Hackert T and Neoptolemos JP: Neoadjuvant and adjuvant chemotherapy in pancreatic cancer. Lang Arch Surg 403(8): 917-932, 2018. PMID: 30397779. DOI: 10.1007/s00423-018-1724-8

24 Versteijne E, Suker M, Groothuis K, Akkermans-Vogelaar JM, Besselink MG, Bonsing BA, Buijsen J, Busch OR, Creemers GM, van Dam RM, Eskens FALM, Festen S, de Groot JWB, Groot Koerkamp B, de Hingh IH, Homs MYV, van Hooft JE, Kerver ED, Luelmo SAC, Neelis KJ, Nuyttens J, Paardekooper GMRM, Patijn GA, van der Sangen MJC, de Vos-Geelen J, Wilmink JW, Zwinderman AH, Punt CJ, van Eijck CH and van Tienhoven G; Dutch Pancreatic Cancer Group: Preoperative chemoradiotherapy versus immediate surgery for resectable and borderline resectable pancreatic cancer: Results of the Dutch randomized phase III PREOPANC Trial. J Clin Oncol 38(16): 1763-1773, 2020. PMID: 32105518. DOI: $10.1200 /$ JCO.19.02274

25 Peng JS, Morris-Stiff G, Ali NS, Wey J, Chalikonda S, El-Hayek KM and Walsh RM: Neoadjuvant chemoradiation is associated with decreased lymph node ratio in borderline resectable pancreatic cancer: A propensity score matched analysis. Hepatobiliary Pancreat Dis Int, 2020. PMID: 32861576. DOI: 10.1016/j.hbpd.2020.08.001

26 Palmer DH, Stocken DD, Hewitt H, Markham CE, Hassan AB, Johnson PJ, Buckels JA and Bramhall SR: A randomized phase 2 trial of neoadjuvant chemotherapy in resectable pancreatic cancer: Gemcitabine alone versus gemcitabine combined with cisplatin. Ann Surg Oncol 14(7): 2088-2096, 2007. PMID: 17453298. DOI: 10.1245/s10434-007-9384-x

27 Tempero MA, Malafa MP, Chiorean EG, Czito B, Scaife C, Narang AK, Fountzilas C, Wolpin BM, Al-Hawary M, Asbun H, Behrman SW, Benson AB, Binder E, Cardin DB, Cha C, Chung
V, Dillhoff M, Dotan E, Ferrone CR, Fisher G, Hardacre J, Hawkins WG, Ko AH, LoConte N, Lowy AM, Moravek C, Nakakura EK, O'Reilly EM, Obando J, Reddy S, Thayer S, Wolff RA, Burns JL and Zuccarino-Catania G: Pancreatic adenocarcinoma, version 1.2019 featured updates to the NCCN guidelines. J Natl Compr Cancer Netw 17(3): 202-210, 2019. PMID: 30865919. DOI: 10.6004/jncen.2019.0014

28 Strijker M, Chen JW, Mungroop TH, Jamieson NB, van Eijck CH, Steyerberg EW, Wilmink JW, Groot Koerkamp B, van Laarhoven HW and Besselink MG: Systematic review of clinical prediction models for survival after surgery for resectable pancreatic cancer. Br J Surgery 106(4): 342-354, 2019. PMID: 30758855. DOI: $10.1002 /$ bjs. 11111

29 Balzano G, Dugnani E, Crippa S, Scavini M, Pasquale V, Aleotti F, Liberati D, Gandolfi A, Belfiori G, Reni M, Doglioni C, Ruffo G, Marmorale C, Falconi M and Piemonti L: A preoperative score to predict early death after pancreatic cancer resection. Dig Liver Dis 49(9): 1050-1056, 2017. PMID: 28734776. DOI: 10.1016/j.dld.2017.06.012

30 Bicakli DH, Uslu R, Güney SC and Coker A: The relationship between nutritional status, performance status, and survival among pancreatic cancer patients. Nutr Cancer 72(2): 202-208, 2020. PMID: 31271302. DOI: 10.1080/01635581.2019.1634217

31 Gilliland TM, Villafane-Ferriol N, Shah KP, Shah RM, Tran Cao HS, Massarweh NN, Silberfein EJ, Choi EA, Hsu C, McElhany AL, Barakat O, Fisher W and Van Buren G: Nutritional and metabolic derangements in pancreatic cancer and pancreatic resection. Nutrients 9(3): 243, 2017. PMID: 28272344. DOI: $10.3390 /$ nu 9030243

32 Stark AP, Sacks GD, Rochefort MM, Donahue TR, Reber HA, Tomlinson JS, Dawson DW, Eibl G and Hines OJ: Long-term survival in patients with pancreatic ductal adenocarcinoma. Surgery 159(6): 1520-1527, 2016. PMID: 26847803. DOI: 10.1016/j.surg.2015.12.024

33 Azizian A, Rühlmann F, Krause T, Bernhardt M, Jo P, König A, Kleiß M, Leha A, Ghadimi M and Gaedcke J: CA19-9 for detecting recurrence of pancreatic cancer. Sci Rep 10(1): 1332, 2020. PMID: 31992753. DOI: 10.1038/s41598-020-57930-x

34 Katz MHG, Varadhachary GR, Fleming JB, Wolff RA, Lee JE, Pisters PW, Vauthey JN, Abdalla EK, Sun CC, Wang H, Crane $\mathrm{CH}$, Lee JH, Tamm EP, Abbruzzese JL and Evans DB: Serum CA 19-9 as a marker of resectability and survival in patients with potentially resectable pancreatic cancer treated with neoadjuvant chemoradiation. Ann Surg Oncol 17(7): 1794-1801, 2010. PMID: 20162463. DOI: 10.1245/s10434-010-0943-1

35 Ikuta S, Sonoda T, Aihara T and Yamanaka N: A combination of platelet-to-lymphocyte ratio and carbohydrate antigen 19-9 predict early recurrence after resection of pancreatic ductal adenocarcinoma. Ann Transl Med 7(18): 461, 2019. PMID: 31700897. DOI: $10.21037 /$ atm.2019.08.35

36 Cloyd JM, Heh V, Pawlik TM, Ejaz A, Dillhoff M, Tsung A, Williams T, Abushahin L, Bridges JFP and Santry H: Neoadjuvant therapy for resectable and borderline resectable pancreatic cancer: A meta-analysis of randomized controlled trials. J Clin Med 9(4): 1129, 2020. PMID: 32326559. DOI: $10.3390 /$ jcm 9041129

Received October 5, 2020

Revised October 28, 2020

Accepted November 4, 2020 